1 Universidade da Califórnia

- Los Angeles (LA), EUA.

Orcid: https://orcid. org/0000-0001-80555441

jmacinko@ucla.edu

2 Universidade Federal do Rio Grande do Sul (UFRGS)

- Porto Alegre (RS), Brasil.

Orcid: https://orcid.

org/0000-0001-6264-

5769

claunara@ghc.com.br

\section{Estratégia Saúde da Família, um forte modelo de Atenção Primária à Saúde que traz resultados}

\author{
The Family Health Strategy, a strong model of Primary Health Care \\ that delivers results
}

James Macinko', Claunara Schilling Mendonça ${ }^{\mathbf{2}}$

DOI: 10.1590/0103-11042018S102

RESUMO Este artigo revisa e sintetiza evidências sobre o impacto da Estratégia Saúde da Família (ESF) a partir do marco conceitual de sistemas de saúde da Organização Mundial da Saúde, o qual agrega os conceitos de acesso, proteção financeira, qualidade dos serviços, eficiência no sistema, impacto na saúde e equidade. Os resultados sugerem que a ESF contribuiu para a melhoria em todos esses indicadores, com alguns efeitos no acesso e equidade com resultados quase chegando aos níveis observados nos países da Organização para Cooperação e Desenvolvimento Econômico. Embora ainda haja bastante espaço para melhorias, a evidência é clara de que a ESF é uma abordagem poderosa e eficaz para a organização da atenção primária à saúde no Brasil.

PALAVRAS-CHAVE Sistema Único de Saúde. Estratégia Saúde da Família. Atenção Primária à Saúde.

\begin{abstract}
This article uses the health systems framework of the World Health Organization to review and synthesize evidence on the impact of the Family Health Strategy (FHS) within the concepts of access, financial protection, quality of services, system efficiency, health impact, and equity. The results suggest that the FHS contributed to the improvement in all those indicators, with effects on access and equity nearly at the level observed in the countries of the Organization for Economic Cooperation and Development. Although there is still great room for improvement, the evidence is clear that the FHS is a powerful and effective approach to the organization of primary health care in Brazil.
\end{abstract}

KEYWORDS Unified Health System. Family Health Strategy. Primary Health Care. 


\section{Introdução}

O futuro do Sistema Único de Saúde (SUS), com maior orientação para atenção primária e capaz de responder às necessidades da população, depende de políticas que atuem sobre todos os níveis de determinação da saúde. Entre elas, a forma como os serviços de saúde estão organizados também atua como um determinante social da saúde e pode contribuir para a melhoria da saúde da população e para a redução de iniquidades, particularmente quando os serviços de atenção primária são explicitamente considerados, ${ }^{\mathbf{1} 2}$. Melhorias nos indicadores de saúde são fundamentais por vários motivos: elas representam um bem em si mesmo, pois pessoas no mundo inteiro valorizam a sua saúde e a saúde dos familiares ${ }^{3}$; a saúde é fundamental para o desenvolvimento individual, pois melhores condições de saúde estão associadas à realização de atividades básicas, como produtividade no trabalho e desempenho acadêmico, entre outros ${ }^{4,5}$. Em nível macro, a ausência de saúde na população (frequentemente medida pela carga de doença) está associada a maiores despesas em saúde e pior desempenho macroeconômico ${ }^{6,7}$. Por esses motivos, os sistemas e serviços de saúde representam um investimento no bem-estar da população, mas o retorno nesse investimento é diferente entre países, que precisam balançar gastos, cobertura, qualidade e equidade, entre outros fatores.

Estudos realizados em países industrializados que avaliam a provisão de serviços de saúde têm demonstrado vantagens quando os sistemas nacionais de saúde são orientados a partir de serviços de atenção primária ${ }^{1,8}$. Um estudo de 31 países (principalmente europeus) evidenciou a complexidade da Atenção Primária à Saúde (APS) e a necessidade de considerar aspectos multidimensionais para avaliar seu impacto 9 . Estudos posteriores nos mesmos 31 países encontraram associação entre a APS forte e melhores indicadores de saúde na população, menores taxas de hospitalizações desnecessárias e menores desigualdades socioeconômicas na saúde ${ }^{10}$, e esse impacto foi ainda maior para pessoas portadores de doenças crônicas ${ }^{11}$. Estudos realizados em países de médio e baixo ingresso mostraram resultados semelhantes, reforçando a importância de investimento adequado na atenção primária12.

O Brasil tem um Sistema Nacional de Saúde, público e universal, denominado SUS, resultado da luta pela redemocratização do País, que traz em seu arcabouço legal importantes elementos como o conceito amplo de saúde, ao considerar os aspectos socioambientais e o entendimento que a saúde é um direito do cidadão e dever do Estado ${ }^{13}$.

Em 2006, foi elaborada e aprovada a Política Nacional de Atenção Básica (PNAB), que explicita a Saúde da Família (SF) como modelo preferencial de reorganização da atenção primária no SUS. Na PNAB, atenção básica é definida como 'um conjunto de ações de saúde desenvolvidas em âmbito individual e coletivo que abrangem a promoção e proteção da saúde, prevenção de agravos, diagnóstico, tratamento, reabilitação e manutenção da saúde’. Essas ações se desenvolvem por meio de uma equipe multidisciplinar, em um território geograficamente definido e com sua respectiva população, tornando-se o primeiro ponto de contato da população com o sistema de saúde. Fortalecem os princípios da APS em um Sistema Universal de Saúde, como é o SUS: universalidade, acessibilidade, coordenação, vínculo, continuidade, integração, responsabilidade, humanização, equidade e participação social14,15. Em 2011, com a proposta de Redes de Atenção à Saúde nas regiões brasileiras, a APS foi definida como porta de entrada do SUS ${ }^{16}$.

Nesse processo histórico, a SF desenvolveu-se de forma gradativa e é a alavanca principal do avanço da APS no Brasil. Nenhuma outra iniciativa dentro do SUS alcançou a magnitude dessa política que hoje é globalmente citada como exemplo de sucesso ${ }^{\mathbf{1 4}}$.

Assim, este artigo pretende apresentar 
uma síntese de alguns dos resultados da Estratégia Saúde da Família (ESF) em relação a vários indicadores de desempenho, derivados dos atributos essenciais da atenção primária (acesso/utilização, cuidados longitudinais, atenção integral) de Starfield ${ }^{\mathbf{8}}$ e do modelo conceitual para avaliação de sistemas de saúde da Organização Mundial da Saúde (OMS) que agrega os conceitos de proteção financeira, qualidade dos serviços, eficiência no sistema, impacto na saúde e equidade ${ }^{15}$.

\section{Métodos}

Este artigo é resultado de uma revisão e síntese da literatura brasileira e internacional sobre os efeitos da ESF no Brasil. Os artigos foram selecionados segundo os seguintes critérios: apresentam os resultados a partir do marco da OMS; medem resultados ou impactos atribuíveis às ações da ESF; os métodos analíticos foram considerados adequados para medir mudanças temporais, controlados os fatores de confusão. A revisão não foi sistemática, porém a apresentação dos resultados favorece artigos considerados metodologicamente rigorosos e relevantes ao marco conceitual da OMS.

Para suplementar a literatura, alguns indicadores foram construídos pelos autores a partir de bancos de dados oficiais de abrangência nacional, incluindo a Pesquisa Nacional por Amostra de Domicílios (PNAD 1998, 2003 e 2008), Pesquisa Nacional de Saúde (PNS) (2013) e o Inquérito Mundial de Saúde (2003). Essas análises são descritivas e, quando necessário, controladas por fatores que poderiam influenciar a interpretação de dados entre populações distintas e entre anos diferentes que incluem idade, sexo, renda familiar, plano de saúde privado e região do País. Todas as análises levam em consideração o delineamento da amostra complexa e incluem pesos analíticos apropriados.

Finalmente, a fim de considerar os desfechos em um contexto global, comparamos alguns dos resultados da ESF com informações de publicações e dados oficiais dos países desenvolvidos pertencentes à Organização para Cooperação e Desenvolvimento Econômico (OCDE) segundo o banco de dados oficial da organização <https://stats.oecd.org/>.

\section{Resultados}

Os efeitos da expansão da ESF têm sido avaliados por um número crescente de estudos divulgados em periódicos nacionais e internacionais, em congressos e outros eventos no Brasil e no exterior. As publicações científicas sobre APS no Brasil nos anos 1990 eram de apenas 1,7 para cada milhão de habitantes, enquanto no Reino Unido era de 51,377. Esse cenário, porém, sofreu uma mudança significativa com o crescimento da ESF, tendo as publicações indexadas com os termos APS/ atenção básica no Brasil crescido a partir da implantação da PNAB, em 2006, como pode ser visto no gráfico 1. Em 2017, o número acumulado de publicações sobre o termo no Brasil alcançou um nível intermediário de publicações (2.475), quando comparado aos outros países listados no gráfico (média = 3.329). 
Gráfico 1. Artigos publicados com os termos 'atenção primária' ou 'atenção básica' no título, por países selecionados, 1980-2017

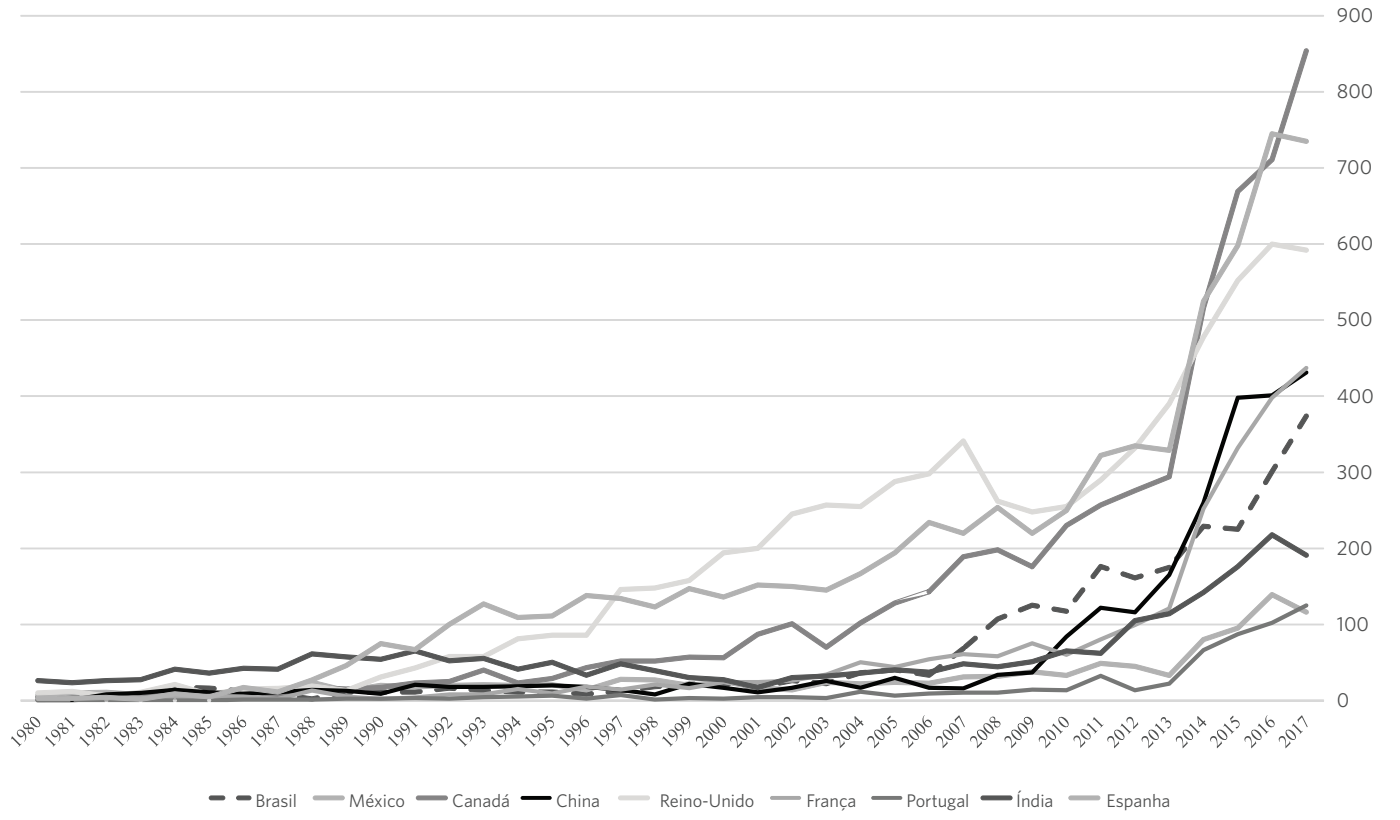

\section{Acesso, utilização e cuidados longitudinais}

Acessibilidade e utilização (acesso realizado) são atributos essenciais da atenção primária porque se a população não acessa os serviços do primeiro nível, não se beneficia de nenhum dos outros atributos dessa modalidade de atenção ${ }^{8}$. Além disso, a atenção primária é mais eficaz quando se concentra na pessoa (e não em uma doença ou órgão, como é o caso das especialidades médicas focais) e cria um vínculo entre o usuário e os profissionais de saúde durante o curso da vida. Por meio desse relacionamento, e do desenvolvimento de registros e outras informações mais qualitativas, os profissionais de APS ganham conhecimento profundo sobre o indivíduo, sua família e a comunidade em que vivem. Os benefícios da longitudinalidade incluem melhor reconhecimento de problemas e necessidades, diagnóstico mais preciso, melhor concordância com os conselhos de tratamento, menos hospitalizações, custos gerais mais baixos, melhor prevenção de alguns tipos de doenças e aumento da satisfação do usuário ${ }^{8,16}$.

A realização de consulta médica nos últimos 12 meses é um indicador de acessibilidade aos serviços de saúde, frequentemente utilizado em comparações internacionais entre sistemas de saúde. Utilizaram-se os dados das PNAD de 1998, 2003 e 2008 e da PNS de 2013 em relação a esse indicador, com análises que controlam para a idade, escolaridade, sexo, renda, doenças crônicas, plano privado e região do País. $\mathrm{O}$ acesso à consulta médica da população brasileira aumentou gradualmente; e, em 2013, foi de 74,2\%, 65,5\% em homens e $82 \%$ em mulheres (tabela 1). Essa proporção de pessoas que realizaram consulta nos últimos 12 meses foi significativamente maior $(\mathrm{p}<0,001)$ por faixa etária mais elevada (84\% para aqueles com 60 anos ou mais) e por doença crônica (94\% para aqueles com uma ou mais doença crônica). 
Tabela 1. Tendências no uso de serviços de saúde, Brasil, 1998-2013

\begin{tabular}{|c|c|c|c|c|c|}
\hline \multirow{2}{*}{ Acesso à consulta médica } & \multicolumn{4}{|c|}{ Anos } & \multirow{2}{*}{ Teste-F1 } \\
\hline & 1998 & 2003 & 2008 & 2013 & \\
\hline Consulta médica ( $<12$ meses) & 54,69 & 62,82 & 67,68 & 74,20 & 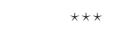 \\
\hline Entre homens & 46,74 & 54,04 & 58,83 & 65,49 & 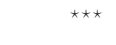 \\
\hline Entre mulheres & 62,33 & 71,18 & 76,07 & 81,97 & 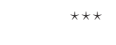 \\
\hline Entre população 60 anos ou mais & 72,18 & 78,00 & 81,33 & 84,55 & 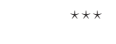 \\
\hline Entre portadores de doenças crônicas² & 76,10 & 82,48 & 85,70 & 88,14 & 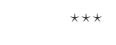 \\
\hline Fonte regular de serviços & 71,22 & 79,27 & 73,64 & 77,07 & ** \\
\hline Entre população 60 anos a mais & 73,79 & 80,35 & 76,62 & 79,48 & 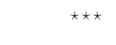 \\
\hline Entre portadores de doenças crônicas ${ }^{2}$ & 75,69 & 82,95 & 78,85 & 80,96 & 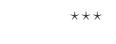 \\
\hline Fonte regular = Posto/centro de saúde & 41,93 & 52,7 & 57,01 & 47,87 & ** \\
\hline Fonte regular = hospital & 34,58 & 27,07 & 21,47 & 21,03 & 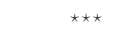 \\
\hline Fonte regular = privado/outro & 23,49 & 20,23 & 21,52 & 31,11 & 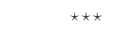 \\
\hline Buscou atenção (<2 semanas) & 12,99 & 14,59 & 14,5 & 17,54 & ** \\
\hline Não conseguiu consulta 1av vez que buscou & 3,68 & 3,59 & 3,75 & 4,75 & * \\
\hline Papanicolau $(<3 \text { anos })^{3}$ & -- & 74,23 & 79,32 & 79,85 & ** \\
\hline Papanicolau, público (<3 anos)3 & -- & 68,46 & 74,71 & 75,76 & 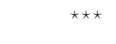 \\
\hline Papanicolau, plano privado $(<3 \text { anos })^{3}$ & -- & 87,97 & 90,16 & 90,48 & * \\
\hline Mamografia $(<2 \text { anos })^{4}$ & -- & 48,01 & 60,56 & 59,92 & ** \\
\hline Consulta odontológica $<2$ anos & 51,76 & 57,83 & 64,93 & 63,69 & $\star \star$ \\
\hline
\end{tabular}

Nota: Os resultados são proporções ponderadas de pesquisas nacionalmente representativas realizadas em 1998, 2003, 2008 e 2013

1. Teste-F para diferença entre os anos ${ }^{\star \star \star}=p<0,001 ;{ }^{* \star}=p<0,01 ;{ }^{*}=p<0.05$

2. Entre aqueles previamente diagnosticados com qualquer condição crônica

3. Entre mulheres de 25 a 59 anos

4. Entre mulheres de 40 a 69 anos (as diretrizes brasileiras mudaram para 50 a 69 anos antes da pesquisa de 2013).

Em termos de barreiras no acesso ao cuidado, entre os $17 \%$ da população que procuraram o serviço de saúde nas duas últimas semanas, $95,25 \%$ receberam atendimento no momento da primeira procura (tabela 1). Esses números são maiores do que os relatados em pesquisas nacionais anteriores e maiores do que os observados nos países da OCDE ${ }^{18}$. Por exemplo, um estudo recente no Reino Unido mostrou que $11 \%$ dos pacientes não puderam obter uma consulta de atenção primária em sua primeira tentativa ${ }^{19}$. No Brasil, entre os $4,75 \%$ que não conseguiram uma consulta, os motivos mais comuns foram falta de médico ou outro profissional de saúde (49\%), incapacidade de marcar consultas ou longos períodos de espera (41\%) e outros motivos, incluindo falta de dinheiro $(9 \%)^{\mathbf{2 0}}$.

Embora o acesso aos cuidados pareça alto, alguns aspectos variam consideravelmente de acordo com as regiões do País. Segundo resultados da PNS 2013, entre pessoas maiores de 60 anos, cujos domicílios foram cadastrados na ESF, a cobertura estadual de visitas domiciliares regulares (pelo menos uma visita a cada 1 ou 2 meses) de Agentes Comunitários de Saúde (ACS) variou 5 vezes, de $17 \%$ no Distrito Federal (DF) para $88 \%$ no estado do Tocantins (TO) (gráfico 2). Assim, embora o acesso à ESF seja facilitado pela localização geográfica das equipes 
de saúde próximas às residências, a ampla variação geográfica da cobertura da ESF por município (e estado) significa exposição desigual a essa forma de atenção à saúde.

Gráfico 2. Proporção de idosos (60+) que relatam receber visitas domiciliares regulares de um Agente Comunitário de Saúde, por unidade federativa brasileira, 2013

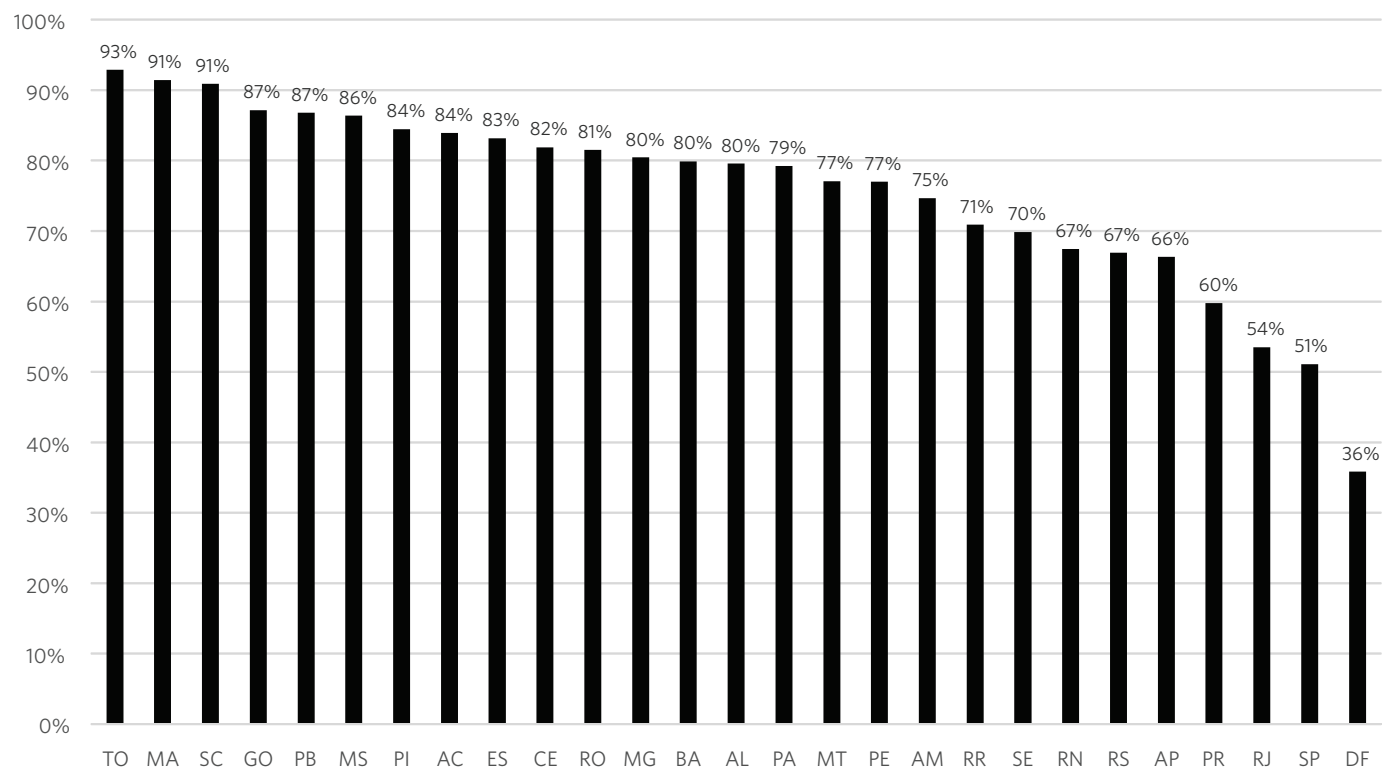

A medida da longitudinalidade costuma ser feita pelo indicador 'fonte usual do cuidado' que mostra se o usuário tem um médico ou serviço que geralmente procura quando precisa de atenção ou conselhos médicos. A tabela 1 também mostra que as tendências desse indicador chegam a $77 \%$ da população em 2013. O período entre 1998 e 2013 teve a mudança mais marcante no tipo de serviço que os brasileiros contam como fonte usual de cuidado, com um declínio entre a proporção que indicou hospital ou pronto-socorro de 35\% em 1998 para 21\% em 2013.

Uma pesquisa analisou o papel da ESF nessa mudança. Segundo os autores, a cobertura da ESF consolidada (em comparação com lugares onde a cobertura ESF foi mais baixa) diminuiu em $37 \%$ a probabilidade de relatar o pronto-socorro ou hospital como fonte usual de cuidados. Quando a população refere uma ou mais doença crônica, a referência à SF como fonte usual do cuidado tem a mesma proporção da população brasileira coberta por plano privado $(80 \%)^{17}$.

Apesar de mostrar uma tendência de aumento, as taxas, ao referir ter uma fonte usual de cuidados no Brasil, são um pouco menores do que as observadas em países da OCDE. Por exemplo, a Pesquisa Internacional de Saúde de 2016 do CommonwealthFund encontrou nos 11 países da OCDE $94 \%$ da população com fonte usual do cuidado, em que a menor proporção foi nos Estados Unidos $(88 \%)$ e a mais alta, na Alemanha $(100 \%)^{21}$.

\section{Integralidade (Comprehensiveness)}

O termo comprehensiveness (cuidados integrais e abrangentes) refere-se à disponibilidade de uma ampla gama de serviços na 
atenção primária e sua provisão adequada em todo o espectro de necessidades da população, exceto para os problemas incomuns, por um provedor de atenção primária. Isso inclui serviços que promovem e preservam a saúde; previnem as doenças, lesões e disfunções; tratam doenças, incapacidades e desconfortos, desde que essas necessidades sejam prevalentes. Os profissionais da atenção primária devem manter a competência em lidar com os problemas prevalentes, que ocorrem em pelo menos dois de cada mil habitantes ao longo de um ano, que os faz buscar os serviços de saúde ${ }^{8}$.

Dos dados da PNS 2013, os adultos com mais de 60 anos cobertos pela ESF que relataram receber cada um de um conjunto de serviços de saúde recomendados para diferentes populações-alvo, os exames comuns, como glicemia e pressão arterial, são realizados em mais de $90 \%$ dos casos (tabela 2). Medidas de colesterol e vacinação contra gripe foram realizadas em mais de $70 \%$ da população. Eletrocardiogramas foram realizados em $66 \%$ dos hipertensos. Apenas dois indicadores tiveram desempenho ruim: o exame de visão geral foi realizado em menos da metade dos usuários idosos acompanhados pela ESF e os exames dos pés entre os diabéticos eram realizados em menos de um terço dos diabéticos idosos usuários da ESF.

Tabela 2. Recebimento de exames e outros serviços, adultos com 60 anos ou mais cobertos pela ESF

\begin{tabular}{lr}
\hline Serviço & $\%$ \\
\hline Exame glicose, <2 anos & 95,7 \\
Pressão arterial, <2 anos & 91,5 \\
Aferição colesterol, <2 anos & 78,6 \\
Vacinação contra gripe, <2 anos & 73,1 \\
Eletrocardiograma (entre hipertensivos) & 66,1 \\
Exame geral de visão, <2 anos & 47,7 \\
Exame de pé (entre diabéticos), <1 ano & 28.7 \\
\hline
\end{tabular}

Fonte: Proporções ponderadas da PNS 2013.

No Brasil, problemas prevalentes em saúde bucal adicionaram investimentos na expansão da saúde bucal no contexto da atenção primária22. O gráfico 3 ilustra as taxas de atendimento odontológico nos últimos 12 meses por idade, quintil de riqueza e ano. A figura demonstra o declínio do uso com a idade e fortes disparidades socioeconômicas. Comparando 1998 a 2013, as taxas absolutas de utilização para todas as faixas etárias aumentaram em $20 \%$, ainda que as disparidades socioeconômicas persistam. Na faixa etária mais jovem, os que estão nos quintis de riqueza mais altos apresentam aproximadamente o dobro da taxa de utilização daqueles no quintil mais baixo. 
Gráfico 3: Probabilidade de pelo menos uma consulta odontológica nos últimos 12 meses, por idade e quintil socioeconômico, Brasil, 1998-2013

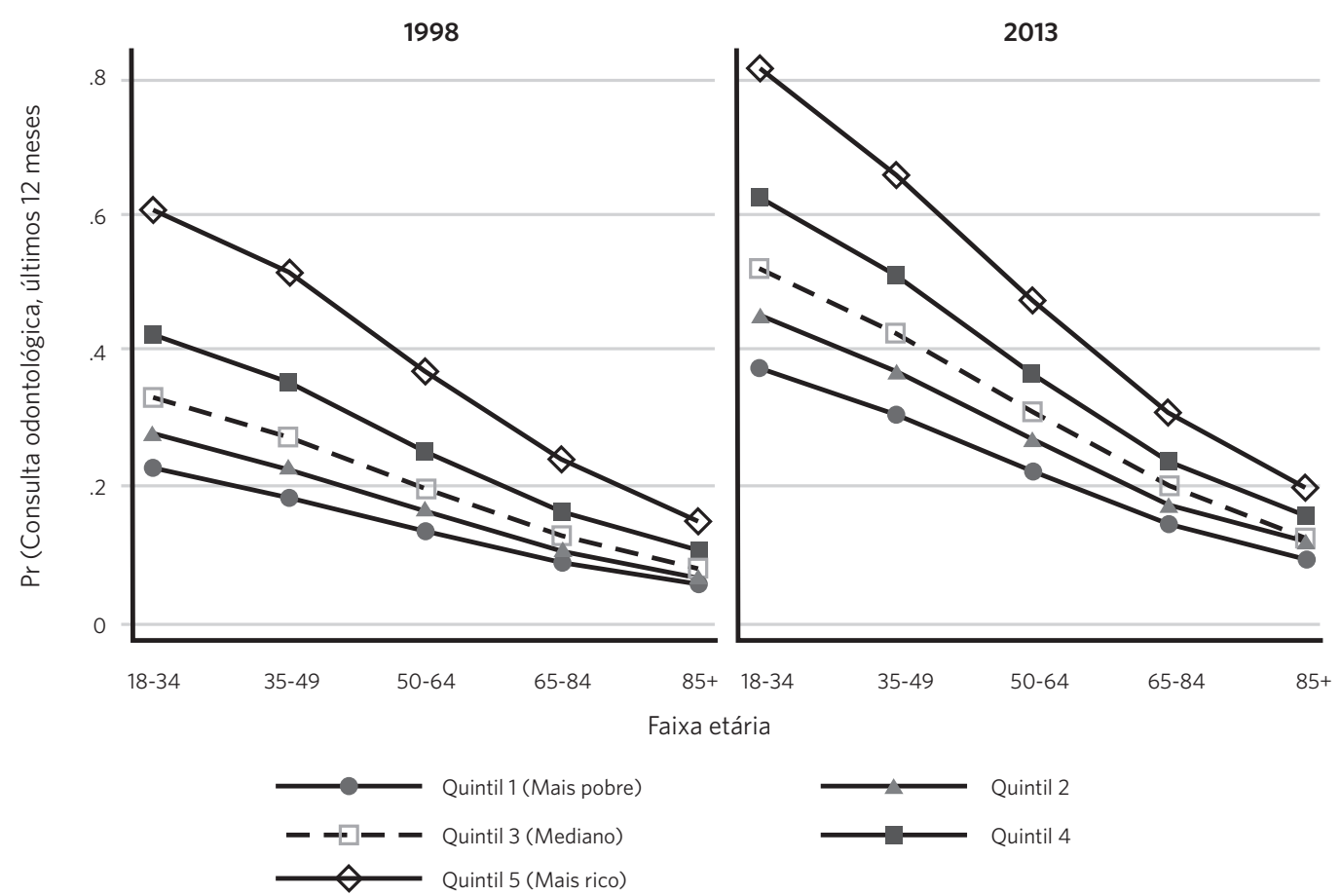

Outra maneira de ampliar os tipos de serviços disponíveis na atenção primária é implantar equipes de saúde multidisciplinares. Em pesquisa sobre trabalhadores comunitários em saúde e impactos positivos do trabalho desses sobre as Metas dos Objetivos de Desenvolvimento do Milênio (ODM), a experiência brasileira de ACS alcançou o melhor resultado, obtendo 34 pontos de um total de 36 , entre 8 países de 3 regiões ${ }^{23}$. Uma revisão sistemática avaliou a efetividade dos ACS no Brasil e encontrou efetividade no cuidado materno-infantil, como tempo do aleitamento materno exclusivo e frequência da avaliação pondero-estatural em crianças; mas baixo nível de evidência para outras ações afeitas ao trabalho dos ACS, como imunização em crianças, tratamento domiciliar observado na tuberculose e o conhecimento de mulheres sobre doenças sexualmente transmissíveis ${ }^{\mathbf{2 4}}$.
Estudos mais recentes encontraram efetividade do trabalho do ACS no controle da dengue $^{25}$ e na promoção da alimentação saudável em menores de 5 anos, após capacitação para melhorar o desempenho dos ACS a fim de responder perguntas da comunidade sobre o tema e identificar erros dietéticos, com melhora do desempenho após a intervenção educativa ${ }^{26}$.

\section{Proteção financeira}

Segundo a OMS, 'Cobertura Universal de Saúde (CUS)' significa que todas as pessoas e comunidades possam usar serviços de saúde (preventivo, curativo, reabilitador e paliativo) de qualidade suficiente para serem eficazes, garantindo que o uso desses serviços não exponha o usuário à pobreza ou a dificuldades financeiras $^{27}$. Apesar das limitações desse conceito, ele 
representa um dos indicadores para as metas de desenvolvimento sustentável28. O SUS garante proteção financeira para todos os serviços oferecidos, mas existem outros custos associados às doenças, por exemplo, na compra de alguns medicamentos, transporte e salários perdidos. Em estudo que comparou os gastos catastróficos em saúde, que representam gastos maiores de $30 \%$ da renda mensal das famílias em países das Américas, com fontes de dados a partir de 2008, o Brasil ocupou a menor posição, com $2,2 \%$ dos domicílios referindo gastos catastróficos ${ }^{29}$.

Em Inquérito do Banco Interamericano de Desenvolvimento (BID), de 2013, que analisa a necessidade de atrasar ou não o comparecimento aos cuidados médicos no último ano por causa dos seus custos, o Brasil apresentou $15,8 \%$ de respostas positivas, estatisticamente semelhante à Nova Zelândia (16,4\%), Colômbia e Holanda (12,6\%), enquanto nos Estados Unidos, a resposta correspondeu a 27,7\%; e no Reino Unido, a 2,1\%21.

Rocha e Soares, em 2010, por meio de análises econométricas, demonstraram efeitos consistentes nas regiões mais pobres do País. A implementação da ESF esteve associada à redução da Mortalidade Infantil (MI), ao crescimento do emprego em adultos, à redução da fertilidade e do espaço entre os filhos e ao aumento de adolescentes na escola e consideram a SF altamente custo-efetiva para essas regiões ${ }^{30}$.

Estudo realizado em Pernambuco, em uma amostra de 785 hipertensos e 823 diabéticos de 35 municípios do estado, avaliou o acesso a medicamentos pela ESF. A oferta de anti-hipertensivos foi de $69 \%$, de hipoglicemiantes orais de $75 \%$ e de insulina e insumos de $65,5 \%$. Entre os hipertensos, 37\% compraram medicamentos; e entre os diabéticos, $30 \%$, com desembolso direto mensal de, em média, $\mathrm{R} \$ 20,00^{31}$.

\section{Qualidade e eficiência}

Embora o SUS tenha facilitado e ampliado o acesso aos serviços de atenção primária de forma bastante satisfatória para os brasileiros, a qualidade desse atendimento tem sido questionada por diversos estudos ${ }^{32-}$ 34. As variações de qualidade resultam de diferenças na disponibilidade de equipamentos básicos, padrões de pessoal e disponibilidade de diferentes profissionais de saúde, má coordenação e disponibilidade de atenção secundária e especializada, variações no gerenciamento e outros apoios institucionais disponíveis para equipes em diferentes municípios ${ }^{33}$. Muitas dessas questões têm sido foco do programa de melhoria da qualidade do governo brasileiro, o Programa de Melhoria do Acesso e Qualidade (PMAQ) ${ }^{35}$.

Em relação à qualidade e à satisfação com serviços de saúde, a tabela 3 apresenta resultados dos inquéritos populacionais de 2003 e 2013, demonstrando um aumento importante na proporção de pessoas que avaliam como boa e muito boa, por exemplo, as explicações dadas pelo médico (91\%); os médicos em geral (89\%); o tempo dado pelo médico para fazer perguntas (85\%); o acolhimento (84,6\%); a limpeza (83,5\%) e o espaço físico (82,7\%). A menor medida de satisfação foi em relação ao tempo de espera, com 61,6\% das pessoas satisfeitas em 2013. Essa proporção melhorou desde 2003 quando era apenas $45,4 \%$. A medida que apresentou piora na satisfação entre 2003 e 2013 foi a disponibilidade de equipamentos e suprimentos, cuja satisfação passou de 87,7\% em 2003 para $78,8 \%$ em 2013, provavelmente em virtude do aumento da expectativa de resposta às necessidades das pessoas na própria APS. 
Tabela 3. Qualidade e satisfação com serviços de saúde, usuários SUS, 2003 e 2013

\begin{tabular}{lccc}
\hline Proporção que avalia como boa / muito boa & $\mathbf{2 0 0 3}$ & $\mathbf{2 0 1 3}$ & $\begin{array}{r}\text { Diferença } \\
\mathbf{2 0 1 3}-\mathbf{2 0 0 3} \mathbf{3}^{1}\end{array}$ \\
\hline Médicos (em geral) & 90,3 & 89,0 & $-1,3+$ \\
Equipamentos e suprimentos disponíveis & 87,7 & 78,9 & $-8,8$ \\
Acolhimento & 86,0 & 84,6 & $-1,4+$ \\
Privacidade adequada & 83,3 & 79,3 & $-4,0$ \\
Médico fornece explicações claras & 80,1 & 91,1 & 11,0 \\
Limpeza das instalações & 79,2 & 83,5 & 4,3 \\
Custos de transporte & 68,8 & 72,0 & 3,2 \\
Médico dá tempo para fazer perguntas & 65,9 & 85,0 & 19,1 \\
Espaço físico suficiente & 63,9 & 82,7 & 18,8 \\
Tempo de espera & 45,4 & 61,6 & 16,2 \\
\hline
\end{tabular}

Fonte: Proporções ponderadas da PMS 2003 e PNS 2013.

Nota: Todas as diferenças são estatisticamente significativas $(p<0,05)$, exceto +

1Valores positivos significam melhoras entre os dois períodos.

Outra medida da qualidade da atenção é a proporção da população-alvo que recebeu exames de rastreamento segundo protocolos nacionais. Em relação à realização do exame de rastreamento do câncer de colo uterino nos últimos três anos, em mulheres de 25 a 64 anos, na PNAD 2003, 68,5\% das mulheres atendidas no SUS realizaram o exame, enquanto entre as mulheres com planos de saúde, $88 \%$ realizaram: uma diferença de $20 \%$. Em 2013, a proporção foi de $75,74 \%$ no público e de $90,5 \%$ no privado, mostrando um aumento em termos absolutos e uma diminuição de desigualdades. Em 2015, nos Estados Unidos, em uma faixa etária mais ampliada, a proporção de mulheres que fizeram o exame foi de $63,8 \%$ entre mulheres sem nenhuma cobertura medica, de $78,4 \%$ entre mulheres com cobertura pública e de $86,8 \%$ entre mulheres com plano privado36.

Um sistema de saúde com APS forte deveria resolver a maioria dos problemas de saúde da população, incluindo controle de doenças crônicas e cuidados de longa duração. Isso pode aumentar a eficiência dos sistemas de saúde pela redução, por exemplo, de hospitalizações desnecessárias, geralmente mais caras para o sistema de saúde que o atendimento na atenção primária. Desde a criação da Lista Brasileira de Internações por Condições Sensíveis (ICSAP) ${ }^{37}$,diversos estudos têm demonstrado associação entre cobertura da SF e redução dessas hospitalizações que podem ser evitadas com bom acesso na atenção primária de boa qualidade. As taxas padronizadas dessas internações variam de acordo com o grau de implantação da SF e da ampliação do acesso e da longitudinalidade, e após um período de redução, pode haver estabilização nas taxas, caso a expansão da cobertura da SF seja sustentada.

Revisão sistemática recente demonstrou que entre os 13 estudos da temática ICSAP avaliados, 8 encontraram redução dessas internações entre $0,7 \%$ e $11 \%$ para cada aumento de $10 \%$ na cobertura da ESF, após controlar os efeitos de outros fatores que influenciam as taxas de hospitalização na população ${ }^{37}$. Apenas um estudo mostrou aumento das internações simultaneamente à expansão da ESF; e, dos estudos que não mostraram efeitos significativos, os autores consideraram que três foram de 
qualidade inferior. Todos os estudos que mostraram diminuição na taxa das ICSAP foram classificados como de alta qualidade ${ }^{37}$.

Outras contribuições da ESF na eficiência do SUS incluem impacto na detecção de casos de doenças tropicais negligenciadas ${ }^{38}$ e melhora na qualidade de estatísticas vitais ${ }^{30,39}$.

\section{Impacto na saúde}

Dos diversos papéis desempenhados pelos sistemas de saúde, dois objetivos tornam-se fundamentais: melhorar a saúde da população e ser equânime na distribuição dos recursos. Vários estudos mostraram que a expansão da ESF tem contribuído de maneira significativa para a redução de várias causas de mortalidade e morbidade no País ${ }^{37}$.

Os resultados mais importantes incluem a contribuição na redução da MI ${ }^{40-43}$. Em 20 anos, a MI no Brasil caiu 61,7\% - de 52,04 mortes por mil nascimentos em 1990 para 19,88 por mil em $2010^{44}$. A expansão da ESF, a partir da década de 1990, teve grande impacto sobre a queda da MI. Além da ampliação do acesso aos serviços de saúde, o aumento na cobertura do pré-natal, da vacinação, a melhoria das condições nutricionais e ambientais, a ampliação de políticas sociais, o aumento no fornecimento de água potável, o incentivo ao aleitamento materno, a ampliação dos bancos de leite humano e da licença maternidade são também apontados como responsáveis por essa redução na $\mathrm{MI}^{\mathbf{4 4}}$.

Até 2018, 18 artigos com metodologias consistentes foram publicados sobre ESF e seu impacto na MI. Desses, $92 \%$ identificaram um impacto significativo na redução da MI ${ }^{45}$. Nesses estudos, os municípios com ESF consolidada ( $>70 \%$ cobertura para 4 anos) experimentaram reduções de taxas de mortalidade neonatal entre $11 \%$ e $44 \%$, mortalidade pós-neonatal entre $17 \%$ e $31 \%$ e mortalidade em menores de 5 anos de $12 \%$ e $13 \% \mathbf{4 1 , 4 3 , 4 6 , 4 7}$.O aumento na cobertura da ESF em $10 \%$ corresponde a uma redução na MI entre $0,4 \%$ e $4,6 \%$, dependendo do período analisado e da unidade de análise (estado, microrregião, ou município) ${ }^{30,40,42,48,49}$.

Outros estudos mostram a imensa flexibilidade da ESF em adaptar-se ao contexto local. Na região do Marajó, no estado do Pará, um arquipélago fluviomarinho de grande vulnerabilidade social, com mais de $60 \%$ da população abaixo da linha da pobreza, em uma população de 62.532 menores de 5 anos, houve redução da mortalidade e das hospitalizações, principalmente por gastroenterites, entre 2011 e 2015, com a ampliação da presença dos médicos na ESF, por meio do Programa Mais Médicos ${ }^{50}$.

O impacto da ESF sobre a saúde da população, porém, não se limita às regiões mais pobres. Seus resultados também têm sido evidenciados em estados como o Rio Grande do Sul, onde a taxa de MI é uma das menores do País. Estudo demonstrou que 10\% de acréscimo na cobertura da SF nesse estado esteve associado à redução de 1\% na MI, de 1994 a $2004^{49}$.

Guanais analisou os efeitos combinados da cobertura da SF com a cobertura do programa de transferência condicionada de renda, Programa Bolsa Família (PBF), entre 1998 e 2010, em 4.583 municípios brasileiros, sobre a MI Pós-Neonatal (MIPN). A redução das taxas de MIPN estavam associadas às maiores coberturas da SF e do PBF, mostrando a importância da combinação de intervenções nas populações mais vulneráveis ${ }^{51}$. Outro estudo avaliou o efeito do PBF e da cobertura da SF nos municípios brasileiros e encontrou efeitos sinérgicos na redução da mortalidade em menores de 5 anos, particularmente nos óbitos atribuídos à baixa renda como as doenças relacionadas a má nutrição e diarreia ${ }^{46}$.

O impacto das políticas sociais, como a SF e o PBF, também foi foco de um estudo de simulação para medir o impacto da emenda à constituição brasileira - EC 95 - que introduziu a mais severa medida de austeridade econômica da modernidade. Esse congelamento dos recursos limitaria a expansão da ESF e a cobertura do PBF, o que, vinculado 
ao aumento da pobreza decorrente da crise econômica, poderá resultar em um aumento da mortalidade de menores de 5 anos, em aproximadamente 20 mil crianças, se esses programas de austeridade forem mantidos ${ }^{52}$.

Estudos sobre a saúde dos adultos nas áreas de cobertura da ESF, a fim de avaliar o monitoramento e controle de pacientes com doenças crônicas, como hipertensão e diabetes, mostram maior acesso da população, principalmente mulheres, a consultas médicas, a medicamentos, mas ainda com importantes deficiências na infraestrutura, nos insumos, na qualificação profissional e no uso das informações para o planejamento ${ }^{53}$. O controle dessas condições tem-se mostrado em menos de $50 \%$ dos casos, como em municípios do estado de Pernambuco com $43,7 \%$ de controle da hipertensão e $30,5 \%$ de controle nos diabéticos ${ }^{54}$.

Em que pese essa situação, a expansão da ESF resultou em redução na taxa de mortalidade e de internações por doenças cardiovasculares e Acidente Vascular Cerebral (AVC) no País. A mortalidade por AVC foi $31 \%$ menor; e por doenças cardiovasculares, foi $36 \%$ menor nos municípios com cobertura da ESF acima de $70 \%{ }^{55}$. Expansão da ESF também foi associada a reduções de mortes por causas consideradas especialmente sensíveis às ações de atenção primária ${ }^{56}$.

Em estudo de base municipal, indivíduos atendidos pela ESF, seis anos após terem tido AVC, tiveram risco de morte $42 \%$ menor que pessoas não vinculadas à ESF. A ESF reduziu a risco absoluto de morte em $16,4 \%{ }^{57}$. A expansão da ESF foi associada a taxas reduzidas de complicações de algumas condições crônicas, como diabetes mellitus ${ }^{58}$.

Em uma amostra de base nacional, de 7.619 idosos de 65 a 74 anos, a fim de avaliar a ESF e a saúde odontológica, encontrou-se efeito protetivo da ESF no sangramento gengival e nas doenças periodontais ${ }^{59}$.Outro estudo mostrou associação com uma redução de disparidades socioeconômicas na utilização dos serviços de saúde bucal22.

\section{Equidade}

Existem consideráveis disparidades de renda e nas condições de vida no Brasil, refletidas por um dos maiores índices de Gini no mundo: 0,51 em 201760. Há também evidências de algumas disparidades socioeconômicas no acesso e no uso de serviços de saúde61,62. Teoricamente, apenas as necessidades de saúde (que incluem necessidades de serviços curativos ou preventivos), e não a posição socioeconômica, deveriam determinar a quantidade de cuidados médicos que uma pessoa utiliza. O Índice de Equidade Horizontal (IEH) mede quanto equitativo é o acesso aos serviços de saúde para determinar se utilização é uma função de necessidade (equitativo) ou de fatores socioeconômicos (inequitativo). $\mathrm{O}$ índice é zero se não existe diferença na utilização entre usuários além das diferenças devidas às necessidades de saúde, positivo quando os ricos usam mais serviços do que necessitam e negativo quando os pobres usam mais ${ }^{63}$. Estudos recentes mostraram um declínio nas desigualdades no uso de serviços de saúde no Brasil. Em 1998, no País, o IEH já mostrou uma orientação pró-pobre para hospitalização ${ }^{64}$. Para consultas médicas e odontológicas, o uso de serviços de saúde é (em graus variados) ainda pró-rico, embora tenha se tornado cada vez mais equitativo ao longo do tempo ${ }^{65}$. Há alguma evidência, entretanto, de que, nos últimos anos, alguns dos ganhos observados na desigualdade relacionada com a saúde podem ter declinado ${ }^{66}$.

Em termos de comparação internacional, a iniquidade em saúde no Brasil parece ser ligeiramente superior à desigualdade encontrada em uma amostra de países da OCDE, mas inferior à maioria dos outros países da América Latina. Um artigo recente usou a PNS 2013 para calcular o IEH para o Brasil e encontrou valores de 0,059 ( 0,23 com ajuste de prevalência) para consultas médicas, 0,165 (0,29 com ajuste de prevalência) para consultas odontológicas e 0,0195 (0,021 com 
ajuste de prevalência) para internações ${ }^{66}$. Em um estudo de 18 países da OCDE, os autores utilizaram apenas o IEH ajustado para prevalência e acharam que, para consultas médicas, esse valor (que foi 0,23 no Brasil) variou de 0,00 no Reino Unido a 0,14 no Canadá e 0,20 nos EUA. Para consultas odontológicas, o IEH foi 0,15 no Reino Unido; e os valores encontrados no Canadá ( 0,3 em 2008) e nos EUA (0,30 em 2009) foram maiores que o valor encontrado no Brasil em 201367.

Na América do Sul, os IEH (não ajustados para prevalência) para consultas médicas eram maiores que os IEH do Brasil: para o Chile e a Colômbia, respectivamente, eram 0,079 e 0,091 para consultas medicas e 0,015 e 0,036 para hospitalizações ${ }^{68,69}$. No entanto, comparar resultados com os de outros países é problemático devido às diferentes medidas de posição socioeconômica utilizadas. Por exemplo, estudos sobre os países da OCDE e sobre o Chile usaram a renda familiar, já estudo sobre a Colômbia utilizou os gastos das famílias.

O gráfico 4 mostra a probabilidade predita de ter pelo menos uma consulta médica nos últimos 12 meses usando dados da PNAD 1998 e PNS 2013. Os dados foram estratificados por três eixos: região do País, quintil de renda familiar e ano. O painel à esquerda representa a situação em 1998. O grupo 1 representa os $20 \%$ da população com renda familiar mais baixa, e teve a probabilidade de realizar uma consulta médica variando entre $50 \%$ para os residentes das regiões mais pobres (Norte e Nordeste) para 55\% para os residentes nas regiões Sul e Sudeste. Essa probabilidade aumenta para cada quintil de renda familiar até o grupo mais rico que teve uma chance de realizar uma consulta médica, de $55 \%$, se mora na região Norte, até $62 \%$, para pessoas que vivem nas regiões mais ricas. As diferenças são marcantes ao relacionar com o painel à direita, que representa a situação em 2013. Nesse ano, a probabilidade de ter uma consulta médica entre as famílias mais pobres, nas regiões mais pobres, foi igual ou superior ao valor para os mais ricos nas regiões mais ricas em 1998. Isso quer dizer que, em termos absolutos, o acesso melhorou para todos os grupos e regiões do País e aumentou ainda mais em termos relativos para as pessoas de média e baixa renda. Os fatores mais consistentemente associados a maior equidade foram a adscrição à ESF e as necessidades de saúde; e os associados a menor equidade, ter planos privados e maior renda ${ }^{65,66}$. 
Gráfico 4: Probabilidade de uma consulta médica nos últimos 12 meses, por renda familiar e região do País, 1998 e 2013

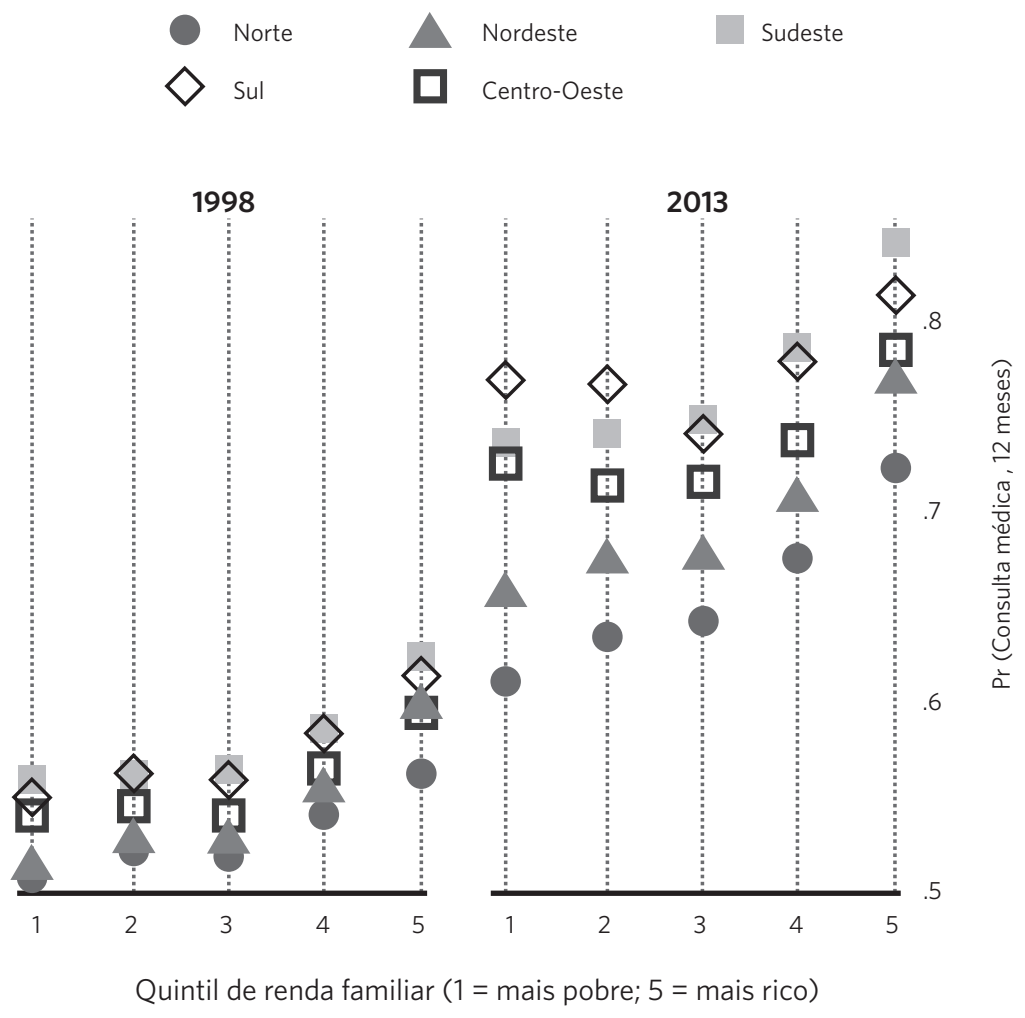

Diante desses desafios, Hone et al. analisaram a associação entre a expansão da ESF e as iniquidades raciais nas mortes evitáveis entre 2000 e $2013^{70}$. A expansão da ESF esteve associada à redução de $15,4 \%$ na mortalidade do grupo da cor negra em relação ao da cor branca. A maior redução foi na mortalidade por doenças infecciosas, deficiências nutricionais e anemias, diabetes e doenças cardiovasculares. Os resultados sugerem que a expansão da ESF reduz iniquidades de mortalidade entre os grupos menos favorecidos na população ${ }^{70}$.

\section{Conclusões}

Este trabalho avaliou e sintetizou as principais evidências sobre a importância e o impacto da ESF no Brasil. Baseado nos resultados apresentados, existem evidências suficientes para concluir que a expansão da ESF teve um impacto muito importante na saúde da população brasileira. Especificamente, a expansão e adequação da ESF facilitou:

- Melhor acesso e utilização de serviços de saúde para a população brasileira e para quem mais precisa - pessoas com menor renda, idosos e portadores de doenças;

- Melhores resultados de saúde incluindo reduções importantes na MI e mortalidade adulta para algumas condições de saúde sensíveis à atenção primária;

- Expansão de acesso a tratamentos, como, por exemplo, os odontológicos, e ampliação no controle de algumas doenças infecciosas; 
- Melhoria na equidade do acesso aos serviços de saúde e diminuição de desigualdades na saúde dos indivíduos;

- Eficiência no SUS devido à redução de hospitalizações desnecessárias e em outras áreas como melhoria na qualidade das estatísticas vitais e sinergias com programas sociais como o PBF e;

- Expansão extensiva de infraestrutura e conhecimento incluindo uma explosão na pesquisa aplicada sobre serviços e sistemas de saúde no Brasil.

Uma limitação importante desta revisão vem do fato de que a maior parte dos estudos aqui apresentados são estudos ecológicos de cortes transversais, fazendo-se necessários estudos complementares mais potentes do ponto de vista das evidências. Existem vários desenhos de estudos robustos em andamento, como a coorte populacional do Estudo Longitudinal de Saúde do Idoso (Elsi) ${ }^{71}$ e coortes sintéticas como as que vêm sendo conduzidas pelo Centro de Integração de Dados e Conhecimentos para Saúde (Cidacs) da Fiocruz. Outras pesquisas estão realizando linkage dos inúmeros bancos de dados com informações individuais, dados de utilização dos serviços e resultados em saúde ao longo do tempo. Além de melhores desenhos analíticos, é necessário ampliar a categorização de qualidade da ESF, para além de sua presença e/ou cobertura, utilizando padrões de qualidade da sua organização, com instrumentos validados para serviços de APS como o PCATool72. Do ponto de vista da avaliação de impacto da política de atenção primária brasileira, por meio da ESF, avaliações de custo-benefício e custo-efetividade e estudos quase-experimentais ainda são necessários.

Como a maioria dos sistemas de saúde em todo o mundo, o SUS luta para atender às necessidades da população, as quais estão em constante evolução. Acelerar as iniciativas de melhoria da qualidade é essencial. Em resposta à necessidade de maior investimento público em saúde, o governo brasileiro lançou, em 2011, um importante modelo de pagamento por desempenho para a ESF a fim de acelerar o investimento na melhoria da infraestrutura e da qualidade técnica do atendimento ${ }^{39}$.

Até o momento, as abordagens mais inovadoras para a organização e provisão de atenção primária (a ESF) concentraram-se nos segmentos mais pobres de muitos municípios. Embora isso tenha levado a melhorias na equidade em saúde, há desafios em alcançar a classe média que prefere buscar serviços no setor privado. Há complicações adicionais decorrentes da natureza descentralizada da gestão da saúde no Brasil, com alguns municípios optando pelo aumento da expansão da ESF, enquanto outros não realizaram investimentos na conversão da UBS tradicional para a ESF mais efetiva.

Juntamente com o aumento da demanda por cuidados de saúde, a rápida expansão da ESF contribuiu para evidenciar a má distribuição de médicos no Brasil, cuja resposta foi o Programa Mais Médicos, contratando mais de 17 mil médicos de outros países ${ }^{73}$. É provável que, à medida que a população continue a envelhecer, a escassez adicional de provedores de saúde - médicos de família, especialistas e equipe de enfermagem apresentará novos desafios, visto que o SUS se esforça para atender às mudanças e necessidades dos cidadãos brasileiros.

Apesar de suas inúmeras conquistas, o SUS enfrenta sérios desafios financeiros e organizacionais. Embora o gasto total com saúde no Brasil seja semelhante à média da OCDE de cerca de $9 \%$ do Produto Interno Bruto, menos da metade desse valor é público, colocando o Brasil muito abaixo da média da OCDE na participação do governo nos gastos com saúde. Atualmente, o SUS enfrenta uma crise importante dada a Emenda Constitucional 95 (EC 95/PEC 55/PEC 241) aprovada em dezembro de 2016 e que limita 
despesas federais em saúde para os próximos 20 anos, congelando os gastos ao nível de 2016 sem incremento além do ajuste pela inflação. A previsão é que essa emenda resultará em um declínio no orçamento da saúde de $\mathrm{R} \$ 415$ bilhões até $2036^{\mathbf{7 4}}$. Reformas adicionais que modificam o financiamento de alguns componentes do SUS (incluindo a ESF $)^{75}$ e a revisão da PNAB podem levar à estagnação ou mesmo à deterioração dos importantes ganhos em saúde obtidos devido a esse modo exitoso de organizar a atenção primária no Brasil.

Em conclusão, observamos que não existe sistema de saúde nem modelo de atenção perfeito, mas, os que apresentam melhores resultados na saúde da população, e maior equidade, são os que têm na APS a centralidade de sua organização. Além disso, existe consenso internacional que redução de investimentos nos sistemas e serviços de saúde pode resultar em piores condições de vida, retrocessos nos avanços já alcançados, mais desigualdades e até desacelerar o crescimento econômico. A ESF cumpre com os requisitos de um bom sistema de APS; e existem muitas evidências sobre sua efetividade. Por isso, é urgente que a ESF (e o SUS) não sofra cortes que interrompam os resultados até aqui encontrados e que passe a ser defendida como uma conquista e um valor ético por toda a sociedade brasileira.

\section{Colaboradores}

Macinko J e Mendonça CS contribuíram para concepção e interpretação dos dados, elaboração do rascunho e revisão crítica do conteúdo e aprovação da versão final do manuscrito.

\section{Referências}

1. Starfield B, Shi L, Macinko J. The Contribution of Primary Care to Health Systems and Health. The Milbank Quarterly. 2005; 83(3):457-502.

2. Bitton A, Ratcliffe HL, Veillard JH, et al. Primary Health Care as a Foundation for Strengthening Health Systems in Low- and Middle-Income Countries. J Gen Intern Med. 2017; 32(5):566-71.

3. Law I, Widdows H. Conceptualising health: insights from the capability approach. Health Care Anal. 2008; 16(4):303-14.
4. Mitchell PM, Roberts TE, Barton PM, et al. Applications of the Capability Approach in the Health Field: A Literature Review. Soc Indic Res. 2017; 133(1):345-71.

5. Black MM, Walker SP, Fernald LCH, et al. Early childhood development coming of age: science through the life course. Lancet. 2017; 389(10064):77-90.

6. Well DN. Accounting for the Effect Of Health on Economic Growth. Q J Econ. 2007; 122(3):1265306. 
7. Bloom DE, Canning D, Sevilla J. The Effect of Health on Economic Growth: A Production Function Approach. World Development. 2004; 32(1):1-13.

8. Starfield B. Primary Care: Balancing Health Needs, Services and Technology. New York: Oxford University Press; 1998.

9. Schafer WL, Boerma WG, Kringos DS, et al. QUALICOPC, a multi-country study evaluating quality, costs and equity in primary care. BMC Fam Pract. 2011; 12:115.

10. Kringos DS, Boerma W, van der Zee J, et al. Europe's strong primary care systems are linked to better population health but also to higher health spending. Health Aff (Millwood). 2013; 32(4):686-94.

11. Hansen J, Groenewegen PP, Boerma WG, et al. Living In A Country With A Strong Primary Care System Is Beneficial To People With Chronic Conditions. Health Aff (Millwood). 2015; 34(9):1531-7.

12. Kruk ME, Porignon D, Rockers PC, et al. The contribution of primary care to health and health systems in low- and middle-income countries: a critical review of major primary care initiatives. Soc Sci Med. 2010; 70(6):904-11.

13. Paim J, Travassos C, Almeida C, et al. The Brazilian health system: history, advances, and challenges. Lancet. 2011; 377(9779):1778-97.

14. Macinko J, Harris M. Brazil's Family Health Strategy: Delivering community based primary care in a universal health system. N Engl J Med. 2015; 372(23):2177-81.

15. Murray CJ, Frenk J. A framework for assessing the performance of health systems. Bull World Health Organ. 2000; 78(6):717-31.

16. Weiss LJ, Blustein J. Faithful patients: The effects of long-term physician-patient relationships on the costs and use of health care by older Americans. Am J Public Health. 1996; (86):1699-700.
17. Dourado I, Medina MG, Aquino R. The effect of the Family Health Strategy on usual source of care in Brazil: data from the 2013 National Health Survey (PNS 2013). Int J Equity Health. 2016; 15(1):151.

18. Macinko J, Lima Costa MF. Access to, use of and satisfaction with health services among adults enrolled in Brazil's Family Health Strategy: evidence from the 2008 National Household Survey. Tropical medicine \& international health: TM \& IH. 2012; 17(1):36-42.

19. Cowling TE, Harris MJ, Majeed A. Access to primary care in England. JAMA Intern Med. 2015; 175(3):467.

20. Boccolini CS, Souza Junior PR. Inequities in Healthcare utilization: results of the Brazilian National Health Survey, 2013. Int J Equity Health. 2016; 15(1):150.

21. Osborn R, Squires D, Doty MM, et al. In New Survey Of Eleven Countries, US Adults Still Struggle With Access To And Affordability Of Health Care. Health Aff (Millwood). 2016; 35(12):2327-36.

22. Peres KG, Peres MA, Boing AF, et al. Reduction of social inequalities in utilization of dental care in Brazil from 1998 to 2008. Rev. Saúde Públ. 2012; 46(2):250-8.

23. Global health Workforce Alliance. Global Experience of Community Health Workers for Delivery of Health Related Millennium Development Goals: A Systematic Review, Country Case Studies, and Recommendations for Scaling Up. Switzerland: Global Health Workforce Alliance (GHWA); April 2010.

24. Giugliani C, Harzheim E, Duncan MS, et al. Effectiveness of community health workers in Brazil: a systematic review. J Ambul Care Manage. 2011; 34(4):326-38.

25. Cazola LH, Tamaki EM, Pontes ER, et al. [The incorporation of activities to control dengue by community health agents]. Rev. Saúde Públ. 2014; 48(1):113-22. 
26. Fernandes MTB, Boscariol SC, Ito TM, et al. Promoção da alimentação saudável do 0 aos 5 anos de idade: a contribuição dos agentes comunitários de saúde. Rev med (Säo Paulo). 2013; 92(2):109-12.

27. Boerma T, Eozenou P, Evans D, et al. Monitoring progress towards universal health coverage at country and global levels. PLoS medicine. 2014; 11(9):e1001731.

28. Abiiro GA, De Allegri M. Universal health coverage from multiple perspectives: a synthesis of conceptual literature and global debates. BMC Int Health Hum Rights. 2015; 15:17.

29. Knaul FM, Wong R, Arreola-Ornelas H, et al. Network on Health F, Social Protection in Latin A, et al. Household catastrophic health expenditures: a comparative analysis of twelve Latin American and Caribbean Countries. Salud Publica Mex. 2011; 53(supl 2):s85-95.

30. Rocha R, Soares RR. Evaluating the impact of community-based health interventions: evidence from Brazil's Family Health Program. Health Econ. 2010; 19(supl):126-58.

31. Barreto MN, Cesse EA, Lima RF, et al. Analysis of access to hypertensive and diabetic drugs in the Family Health Strategy, State of Pernambuco, Brazil. Rev Bras Epidemiol. 2015; 18(2):413-24.

32. Facchini LA, Piccini RX, Tomasi E, et al. Desempenho do PSF no Sul e no Nordeste do Brasil: avaliação institucional e epidemiológica da Atenção Básica à Saúde. Ciênc Saúde Colet. 2006; 11(3):669-681.

33. Facchini LA, Piccini RX, Tomasi E, et al. [Evaluation of the effectiveness of Primary Health Care in South and Northeast Brazil: methodological contributions]. Cad. Saude Pública. 2008; 24(supl 1):S15972.

34. Fausto MC, Bousquat A, Lima JG, et al. Evaluation of Brazilian Primary Health Care From the Perspective of the Users: Accessible, Continuous, and Acceptable? J Ambul Care Manage. 2017;40(Supl
2 Supplement, The Brazilian National Program f):S60-S70.

35. Macinko J, Harris MJ, Rocha MG. Brazil's National Program for Improving Primary Care Access and Quality (PMAQ): Fulfilling the Potential of the World's Largest Payment for Performance System in Primary Care. J Ambul Care Manage. 2017;40 (Supl 2 Supplement, The Brazilian National Program for Improving Primary Care Access and Quality - PMAQ):S4-S11.

36. White A, Thompson TD, White MC, et al. Cancer Screening Test Use - United States, 2015. MMWR Morb Mortal Wkly Rep. 2017; 66(8):201-6.

37. Alfradique ME, Bonolo PF, Dourado I, et al. [Ambulatory care sensitive hospitalizations: elaboration of Brazilian list as a tool for measuring health system performance (Project ICSAP--Brazil)]. Cad. Saude Pública. 2009; 25(6):1337-49.

38. Nery JS, Pereira SM, Rasella D, et al. Effect of the Brazilian conditional cash transfer and primary health care programs on the new case detection rate of leprosy. PLoS neglected tropical diseases. 2014; 8(11):e3357.

39. Rasella D, Aquino R, Barreto ML. Impact of the Family Health Program on the quality of vital information and reduction of child unattended deaths in Brazil: an ecological longitudinal study. BMC public health. 2010; 10:380.

40. Macinko J, Guanais F, Souza F. An Evaluation of the Impact of the Family Health Program on Infant Mortality in Brazil, 1990-2002. Journal of Epidemiology and Community Health. 2006; 60:13-9.

41. Aquino R, Oliveira NF, Barreto ML. Impact of the Family Health Program on Infant Mortality in Brazilian Municipalities. Am J Public Health. 2009; 99(1):87-93.

42. Macinko J, Marinho SMF, Guanais FC, et al. Going to scale with community-based primary care: an analysis of the family health program and infant 
mortality in Brazil, 1999-2004. Soc Sci Med. 2007; 65(10):2070-80.

43. Rasella D, Aquino R, Barreto ML. Reducing Childhood Mortality From Diarrhea and Lower Respiratory Tract Infections in Brazil. Pediatrics. 2010; 126(3):e534-40.

44. Countdown Working Group on Health Policy and Health Systems. Assessment of the health system and policy environment as a critical complement to tracking intervention coverage for maternal, newborn, and child health. Lancet. 2008; 371(9620):1284-93.

45. Bastos ML, Menzies D, Hone T, et al. The impact of the Brazilian family health strategy on selected primary care sensitive conditions: A systematic review. PLoS One. 2017; 12(8):e0182336.

46. Rasella D, Aquino R, Santos CA, et al. Effect of a conditional cash transfer programme on childhood mortality: a nationwide analysis of Brazilian municipalities. Lancet. 2013; 382(9886):57-64.

47. Brentani A, Grisi S, Taniguchi MT, et al. Rollout of community-based family health strategy (programa de saude de familia) is associated with large reductions in neonatal mortality in Sao Paulo, Brazil. SSM Popul Health. 2016; 2:55-61.

48. Guanais FC, Macinko J. The health effects of decentralizing primary care in Brazil. Health Aff (Millwood). 2009; 28(4):1127-35.

49. Zanini RR, Moraes AB, Giugliani ER, et al. Infant mortality trends in the State of Rio Grande do Sul, Brazil, 1994-2004: a multilevel analysis of individual and community risk factors. Cad. Saude Pública. 2009; 25(5):1035-45.

50. Carneiro VB, Oliveira PTR, Bastos MSCB, et al. Avaliação da mortalidade e internações por condição sensível à atenção primária em menores de 5 anos, antes e durante o programa mais médicos, no Marajó-Pará-Brasil. Saúde em Redes. 2016; 2(4):360-71.
51. Guanais FC. The combined effects of the expansion of primary health care and conditional cash transfers on infant mortality in Brazil, 1998-2010. Am J Public Health. 2013; 103(11):2000-6.

52. Rasella D, Basu S, Hone T, et al. Child morbidity and mortality associated with alternative policy responses to the economic crisis in Brazil: A nationwide microsimulation study. PLoS medicine. 2018; 15(5):e1002570.

53. Zavatini MA, Obreli-Neto PR, Cuman RK. [Family health strategy in the treatment of chronic-degenerative diseases: achievements and challenges]. Rev Gaucha Enferm. 2010; 31(4):647-54.

54. Fontbonne A, Cesse EA, Sousa IM, et al. Risk factor control in hypertensive and diabetic subjects attended by the Family Health Strategy in the State of Pernambuco, Brazil: the SERVIDIAH study. Cad. Saúde Pública. 2013; 29(6):1195-204.

55. Rasella D, Harhay MO, Pamponet ML, et al. Impact of primary health care on mortality from heart and cerebrovascular diseases in Brazil: a nationwide analysis of longitudinal data. Bmj. 2014; 349:g4014.

56. Hone T, Rasella D, Barreto M, et al. Large Reductions In Amenable Mortality Associated With Brazil's Primary Care Expansion And Strong Health Governance. Health Aff (Millwood). 2017; 36(1):149-58.

57. Cabral NL, Franco S, Longo A, et al. The Brazilian Family Health Program and secondary stroke and myocardial infarction prevention: a 6-year cohort study. Am J Public Health. 2012; 102(12):e90-5.

58. Klafka A, Duncan B, Rosa R, et al. Mortality from acute complications of diabetes mellitus in Brazil, 2006-2010. Epidemiol Serv Saúde. 2014; 23(3):45562.

59. Dalazen CE, De Carli AD, Bomfim RA, et al. Contextual and Individual Factors Influencing Periodontal Treatment Needs by Elderly Brazilians: A Multilevel Analysis. PLoS One. 2016; 11(6):e0156231. 
60. World Bank Open Data. GINI index. World Bank, Development Research Group. Data are based on primary household survey data obtained from government statistical agencies and World Bank country departments[internet]. [acesso em 2018 ago 16]. Disponível em: https://data.worldbank.org/. World Bank Group. 2018.

61. Lima-Costa MF, Loyola Filho AI, Matos DL. [Trends in health conditions and use of health services by the Brazilian elderly: a study based on the National Household Sample Survey $(1998,2003)]$. Cad. Saude Publica. 2007; 23(10):2467-78.

62. Travassos C, Viacava F, Pinheiro R, et al. [Utilization of health care services in Brazil: gender, family characteristics, and social status]. Rev Panam Salud Publica. 2002; 11(5-6):365-73.

63. Wagstaff A. The bounds of the concentration index when the variable of interest is binary, with an application to immunization inequality. Health Econ. 2005; 14(4):429-32.

64. Macinko J, Lima-Costa MF. Horizontal equity in health care utilization in Brazil, 1998-2008. Int J Equity Health.2012; 11:33.

65. Almeida G, Sarti FM. Measuring evolution of income-related inequalities in health and health care utilization in selected Latin American and Caribbean countries. Rev Panam Salud Publica. 2013; 33(2):83-9.

66. Mullachery P, Silver D, Macinko J. Changes in health care inequity in Brazil between 2008 and 2013. Int J Equity Health. 2016; 15(1):140.

67. Devaux M. Income-related inequalities and inequities in health care services utilisation in 18 selected OECD countries. Eur J Health Econ. 2015; 16(1):2133.

68. Vasquez F, Paraje G, Estay M. Income-related inequality in health and health care utilization in Chi- le, 2000-2009. Rev Panam Salud Publica. 2013; 33(2):98-106.

69. Ruiz Gomez F, Zapata Jaramillo T, Garavito Beltran L. Colombian health care system: results on equity for five health dimensions, 2003-2008. Rev Panam Salud Publica. 2013; 33(2):107-15.

70. Hone T, Rasella D, Barreto ML, et al. Association between expansion of primary healthcare and racial inequalities in mortality amenable to primary care in Brazil: A national longitudinal analysis. PLoS medicine. 2017; 14(5):e1002306.

71. Lima-Costa MF, Andrade FB, Souza PRB, et al. The Brazilian Longitudinal Study of Aging (ELSI-Brazil): Objectives and Design. Am J Epidemiol. 2018; 187(7):1345-53.

72. Brasil. Ministério da Saúde. Secretaria de Atenção em Saúde. Departamento de Atenção Básica. Manual do instrumento de avaliação da atenção primária à saúde: Primary Care Assessment Tool PCAtool-Brasil. Brasília, DF: Ministério da Saúde; 2010.

73. Kemper ES, Mendonca AV, Sousa MF. The Mais Médicos (More Doctors) Program: panorama of the scientific output. Ciênc Saúde Colet. 2016; 21(9):2785-96.

74. Doniec K, Dall'Alba R, King L. Austerity threatens universal health coverage in Brazil. Lancet. 2016; 388(10047):867-8.

75. Massuda A, Hone T, Leles FAG, et al. The Brazilian health system at crossroads: progress, crisis and resilience. BMJ Glob Health. 2018; 3(4):e000829.

Recebido em 23/08/2018

Aprovado em 26/08/2018

Conflito de interesses: inexistente

Suporte financeiro: não houve 